\title{
Intravascular Leiomyomatosis and the CT Appearance: A Case Report and Review of Literature
}

\author{
Shanrong Shu, Ruiman $\mathrm{Li}^{*}$, Xuesong Gao \\ Department of Gynecology and Obstetrics, the First Affiliated Hospital of Jinan University, Guangzhou, People's Republic of China
}

Email address:

liruimanssr@163.com (Ruiman Li)

${ }^{*}$ Corresponding author

To cite this article:

Shanrong Shu, Ruiman Li, Xuesong Gao. Intravascular Leiomyomatosis and the CT Appearance: A Case Report and Review of Literature. Clinical Medicine Research. Vol. 6, No. 4, 2017, pp. 135-138. doi: 10.11648/j.cmr.20170604.14

Received: May 4, 2017; Accepted: June 14, 2017; Published: July 4, 2017

\begin{abstract}
Intravenous leiomyomatosis (IVL) is a benign smooth muscle tumor which may cause fatal outcome. Furthermore, the clinical manifestations are variable and nonspecific, typically resulting in misdiagnosis. Computerized Tomography (CT) played a vital role in the diagnostic process and pre-surgical assessment. Here we describe one case of IVL extending from the inferior vena cava to the right atrium and ventricle, then review the literature and discuss the imaging findings and clinical manifestation of this tumor. It is suggestive for clinicians to be aware of this condition and highly suspect a middle-aged woman with a history of uterine leiomyoma presenting with an inferior vena cava filling defect or mass shadow to early diagnose and treat.
\end{abstract}

Keywords: Intravenous Leiomyomatosis, Computerized Tomography, Clinical Manifestation

\section{Introduction}

Intravenous leiomyomatosis (IVL) is a rare histologically benign tumor originated in the uterine mesoderm, but it is behaviorally malignant, potentially life threatening tumor with unpredictable biological behavior, due to growing and extension with the intrauterine venous system. Birch-Hirschfeld firstly descripted this tumor in German in 1895 [1]. Until 80 years later, Norris [2] suggested using intravenous leiomyomatosis as the diagnostic name of this disease in 1975. Smooth muscle cell spreading along blood vessel like nodule is the main manifestation. Though the organizational structure of the tumor is the hyperplasia of smooth muscle cell, which is similar to leiomyoma of uterus, it is completely different from uterine leiomyoma considering it's malignant growth way [3]. $10 \% \sim 30 \%$ of affected patients has complication in postcava, right atrium and lung, and it may cause death in some severe circumstance [4]. Due to its rarity and the non-specific clinical manifestations, IVL is frequently misdiagnosed as malignant thrombus or thrombosis. Thus, patients suffering from IVL were often treated inappropriately. To explore the related clinical manifestation and useful examination is the focus of clinicians. Here we report a case of IVL with large vascular involvement and the appearance of Computerized Tomography (CT) in the chest, through which clinicians can get some useful information.

\section{Case Description}

A 46-years old female patient was admitted in our hospital, presenting with a 4-day history of palpitations and shortness of breath that were exacerbated upon exertion. The patient also suffered from hypertension $(150 / 110 \mathrm{mmHg})$ with dizziness, headache, decreased vision, paroxysmal nocturnal dyspnea, which could not be alleviated by antihypertensive drugs. Physical examination revealed slight bilateral leg edema and no other abnormalities. Echo- cardiography showed a mobile, solid mass in the right atrium. The lesion, which almost blocked the tricuspid valve, was initially diagnosed as atrial myxoma or thrombus. The serum levels of the tumor marker such as CEA/CA125/CA199 and other blood or bio-chemical markers were within the normal range. The patient's medical history included a myomectomy with no other notable family history.

The abdominal CT scan was performed without or with contrast media. On the scan by $\mathrm{CT}$, a mass shadow was observed in the right atrium, which extended to inferior vena 
cava (IVC) with a soft tissue density of 18-24 HU on plain images and 30-42 HU on contrast-enhanced images. The lesion stretched into the right ventricle through the tricuspid valve, and stretched as far as the right ciliac vein and internal iliac vein, As shown in Figure.
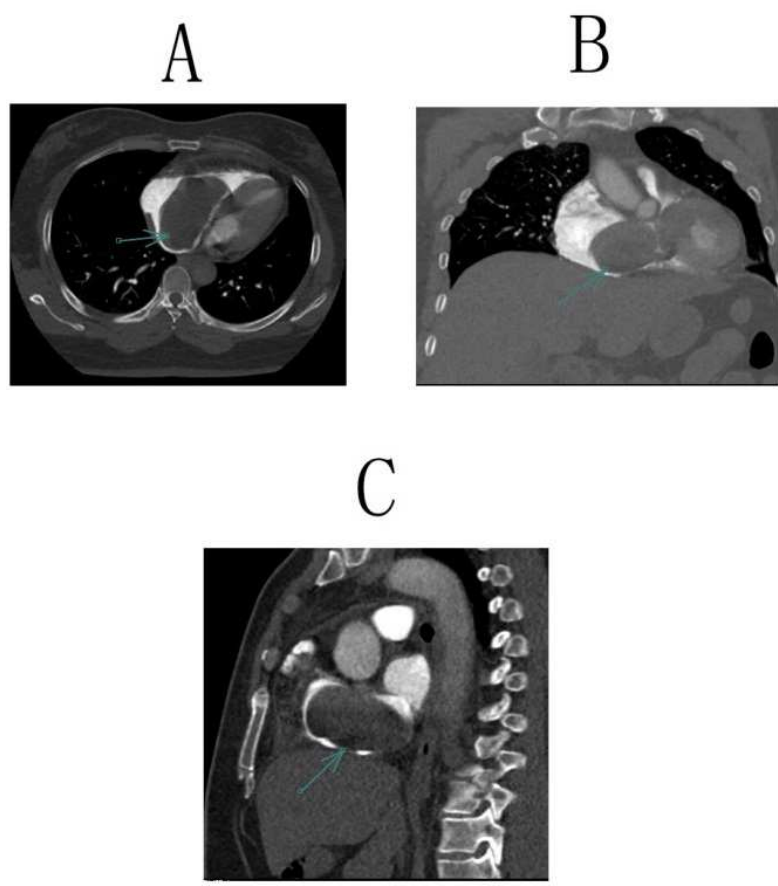

Figure 1. CT scan show a mass shadow in the heart. A. the mass was shown in transverse section. B. the mass was shown in coronal plane. C. the mass was shown in sagittal section.

Considering the imaging findings, completed surgical excision of the tumor in the IVC was performed carefully through right atriotomy. The tumor had no stalk or obvious adhesion with the wall of the right atrium. The resected gross specimen was a yellowish-white tumor with a smooth border and a tip. The tumor appeared as a sausage-shaped protrusion with a maximum diameter of $10 \mathrm{~cm}$. Immunohistochemical analysis using monoclonal antibodies revealed the positive rate of $\mathrm{Ki}-67 / \mathrm{ER} / \mathrm{PR}$ were $5 \%, 80 \%$ and $90 \%$ respectively. The expression of smooth muscle actin (SMA) and desmin were positive, and the expression of CD117/CD34/ DOG-1/S-100 were negative.

\section{Discussion}

\subsection{Etiology and Pathogenesis}

IVL is a rare benign tumor that is histologically characterized by nodular masses of smooth muscle cells growing within systemic veins. It typically originates from the uterus and may involve ovarian veins. Occasionally, the tumor occurs in other intravascular locations, distant from the uterus. The tumors were described as nodular or spiral within pelvic veins and the IVC, or extending to the right cardiac chambers and bilateral pulmonary arteries. The patho- physiology of IVL is not clearly established, but two theories have been proposed. One theory suggests leiomyomatosis originating in invasive uterine fibroids, and then invading along venous lumne [5]. Another theory insists tumor deriving directly from smooth muscle with vascular disease in uterine [6]. Leiomyomatosis stems from uterine, and spreads along one side of the venous system, and extends from there to the inferior vena cava. In some cases, it may reach the right atrium, ventricle, or pulmonary artery. It is reported that $10 \% \sim 30 \%$ of IVL enters the right side of the cardiac chamber through the inferior vena cava [4]. This disease is classified into four phrases according to the location of affiliated lesions. When the lesions locate in uterine and parametrium, it is phase I. When the lesions locate in pelvic vein, it is phase II. When the lesions situate in inferior vena cava, it is phase III, and when the lesions situate in heart cavity, it is phase IV. In this case, the disease was phase IV.

\subsection{Clinical and Pathological Feature}

Literatures reported that $[7,8]$ the peak incidence of VIL ranged from 35 to 63 years old, mostly occurred in childbearing aged women with the history of myoma of uterus. Clinical manifestations are various and nonspecific. Patients may be asymptomatic with IVL discovered only incidentally, or IVL may present with symptoms that mirror the distribution of IVL extension. For example, patients with inferior vena cava occlusion may have lower extremity edema, and abdominal swelling, whereas those with intracardiac obstruction may have dyspnea, chest pain, congestive heart failure, or even sudden death [9]. The clinical signs and symptoms predominantly depended on the degree of intravascular obstruction caused by the tumor. When the lesion was focused on iliac vein and inferior vena cava, the symptom was slight or no specific symptom. When the right atrium or right ventricle were affiliated, the disease could cause cardiac insufficiency leading to chest tightness, short of breath, syncope and sudden death. In pathologic examination, we found the leiomyoma cells were spirally arranged [10]. Meanwhile, ER and PR were expressed in the cells, which suggested the disease was dependent on estrogen and progesterin hormone [11]. In our case, the clinic manifestation and pathologic examination were in accord with literature reports.

\subsection{CT Expression}

CT scan is one of the most useful imaging modalities for the diagnosis of IVL. The high-density resolution and multiplanar capability of CT render this modality favorable for characterizing the continuity of intraluminal tumors. Jin X [12] reported one case of IVL, CT examination showed a soft tissue mass shadow in the left pelvic cavity. There are filling defect and soft tissue mass shadow in the left iliac vein/ common iliac vein/ inferior vena cava/ the right atrium and right ventricle. Nogales FF [13] reported seven cases of IVL. Among the reported seven cases, 2 cases demonstrated the uterine was increases with multiple nodules, 4 cases showed soft tissue mass shadow in internal iliac vein/postcava/ right atrium, 2 cases suggested the proximal renal vein was 
affiliated, 1 case showed filling defect existing in hepatic right lower veins, 2 cases showed enhancement in the periphery of tumor, and 3 cases showed well-circumscribed and tangled mass of vessels across tumor. Magyar E' [14] research proposed that the mass in right atrium was the main imaging expression of IVL, which appeared uneven enhancement after the enhanced scans.

According to the above literature reports and our case, we can conclude that the CT manifestation of IVL has specific features such as uterine or parametrium mass shadow, filling defect or mass shadow in iliac vein and inferior vena cava, sometimes the mass extends to the right atrium or the right ventricle with some mobility and uneven/even enhance- ment [15]. Also, the mass can involve the renal or hepatic vein, sometime the tortuous and expanded vein can be seen in pelvic cavity (Considering the formation of collateral circulation) [16]. For those patients, if we take Computerized Tomographic Angiography (CTA), the correlation of blood vessel wall with the mass in iliac vein/inferior vena cava/ right cardiac cavity can be reconstructed with Magnetic Resonance Imaging (MRI), by which we can multidimensionally observe the mass in pelvic cavity and the collateral circulation.

\subsection{Differential Diagnosis}

Upon noting an intravascular mass within the inferior vena cava, differential diagnoses include intravenous thrombosis, right atrial myxoma, leiomyosarcoma, malignant tumor embolus, and intravenous leiomyoma, as these entities have similar CT or MRI characteristics to IVL. Detailed preoperative imaging may help to reveal the underlying lesion, except in the case of leiomyosarcoma, which is challenging in the early stages of the disease, however, when leiomyosarcoma presents with visible infltration and invasion around structures, diagnosis becomes more simple [17]. Meanwhile, histopathology is required for definitive diagnosis as these tumors appear radiographically indistinct. Intravenous thrombosis appear similar to IVL on CT scan or pelvic ultrasound but differ in that they do not enhance after administration of gadolinium-based contrast with CT. Right atrial myxoma typically involves the cardiac chamber and does not normally extend into the IVC. The features of malignant thrombosis are similar to those of the primary tumor and the lesion is generally considerably smaller than the IVL. In addition, malignant thrombosis is not associated with a history of uterine myoma or surgery for uterine myoma [18]. Complete surgical excision of the tumor is an appropriate treatment option for IVL, and the final diagnosis is dependent on histopathological analysis.

\subsection{Treatment and Prognosis}

The treatment of choice is radical surgical resection of the entire lesion, which need a multidisciplinary team to remove the pelvic organs such as uterus, fallopian tubes, ovaries, and any parametrial leiomyoma, also including intravascular tumor, and any intracardiac components. Whether to take one-stage or two-stage operation to separate chest surgery from abdominal- pelvic surgery depends on the condition of patients. Wang et al [19] reported on 30 patients treated for
IVL at a single center, the largest known series to date, and found that patients with a good performance status and minor or no intracardiac extension can safely undergo a single-stage operation. If the patients have serious complication, it is better to two-stage operation.

\section{Conclusion}

IVL has some specific clinical and imaging characteristics. It is possible for us to diagnose this disease in the early stage, if we are familiar with the history of the patients, the pathogenetic process and the CT manifestation. CT examination can provide us with the range of lesions, the adhesive degree of the vessel wall and the situation of collateral circulation. All of which is helpful for surgical operation.

\section{Acknowledgements}

This work was supported by Natural Science Foundation of China (81402143) and National College Students Innovation and Entrepreneurship Training Program (201610559019).

\section{References}

[1] FV B. Text book of Pathological Anatomy. 5th edition. F. C. W. Vogel, Leipzig, 1896. 226-258.

[2] Norris H J, Parmley T. Mesenchymal tumors of the uterus. V. Intravenous leiomyomatosis. A clinical and pathologic study of 14 cases. Cancer. 1975; 36 (6): 2164-2178.

[3] Rajaii-Khorasani A, Kahrom M, Hashemzadeh M, Tayebi S, Ghazi M. Hamedanchi A. Pulmonary artery extension of uterine leiomyoma. J Card Surg. 2012; 27 (4): 466-469.

[4] Lee S, Kim DK, Narm KS, Cho SH. Pulmonary artery emboli zation of intravenous leiomyo matosis extending into the right atrium. Korean J Thorac Cardiovasc Surg. 2011; 44 (3): 243-246.

[5] Valdes DV, Conley CR, Stone WM, Collins JM, Magrina JF. Update on intravenous leiomyo matosis: report of five patients and literature review. Eur J Obstet Gynecol Reprod Biol. 2013; 171 (2): 209-213.

[6] Izzat MB, Bayazid SS, huaibi I. Pelvic intravenous leiomyo matosis with transcaval extension to the heart and pulmonary arteries. J Card Surg. 2011; 26 (6): 630-631.

[7] Du J, Zhao X, Guo D, Li H Sun B. Intravenous leiomyomatosis of the uterus: a clinicopathologic study of 18 cases, with emphasis on early diagnosis and appropriate treatment stra tegies. Hum Pathol. 2011; 42 (9): 1240-1246.

[8] Clay TD, Dimitriou J, McNally OM, Russell PA, Newcomb AE, Wilson AM. Intravenous leiomyo matosis with intracardiac extension- a review of diagnosis and management with an illustrative case. Surg Oncol. 2013; 22 (3): e44-e52.

[9] Matos AP, Ramalho M, Palas J, Heredia V. Heart extension of an intravenous leiomyomatosis. Clin Imaging. 2013; 37 (2): 369-373. 
[10] Bender LC, Mitsumori LM, Lloyd KA, Stambaugh LR. AIRP best cases in radiologic-pathologic correlation: intravenous leiomyomatosis. Radiographics. 2011; 31 (4): 1053-1058.

[11] Barksdale J, Abolhoda A, Saremi F. Intravenous leiomyo matosis presenting as acute Budd-Chiari syndrome. J Vasc Surg 2011; 54 (3): 860-863.

[12] Nogales FF, Navarro N, Martinez D VJ, Contreras F, Redondo $\mathrm{C}$, Herraiz MA, et al. Uterine intravascular leiomyomatosis: an update and report of seven cases. Int J Gynecol Pathol. 1987; 6 (4): $331-339$

[13] Magyar E, Salamon F, Tomcsanyi I. Intravascular leiomyo matosis presenting as a right atrial mass. Lege Artis Med. 2007; 17 (10): 694-697.

[14] Gunderson CC, Parsons B, Penaroza S, Peyton MD, Landrum LM. Intravenous leiomyomatosis disguised as a large deep vein thrombosis. J Radiol Case Rep. 2016; 10 (5): 29-35.
[15] Barksdale J, Abolhoda A, Saremi F. Intravenous leiomyoma tosis presenting as acute Budd-Chiari syndrome. J Vasc Surg. 2011; 54 (3): 860-863.

[16] Lu CZ, Cao SS, Wang W, Liu J, Fu N, Lu F. Usefulness of PET/ $\mathrm{CT}$ in the diagnosis of recurrent or metastasized differentiated thyroid carcinoma. Oncology letters. 2016; 4 (11): 2420-2423.

[17] McDonald DK, Kalva SP, Fan CM, Vasilyev A. Leiomyo sarcoma of the uterus with intravascular tumor extension and pulmonary tumor embolism. Cardiovasc Intervent Radiol. 2007; 30 (1): 140-142.

[18] Osawa H, Hosaka S, Akashi O, Furukawa H, Egi K. A case of intravenous leiomyomatosis of uterine origin, extending throughthe inferior vena cava to right atrium. Gen Thorac Cardiovasc Surg. 2013; 61 (2): 104-107.

[19] Wang J, Yang J, Huang H, Li Y, Miao Q, Lu X, et al. Manage ment of intravenous leiomyomatosis with intracaval and intra cardiac extension. Obstet Gynecol 2012; 120 (6): 1400-1406. 